\title{
A novel, non-invasive, integral imaging assessment in atrial fibrillation by cardiac tomography
}

\author{
Un nuevo método no invasivo en la valoración integral de la fibrilación auricular por \\ tomografía cardíaca
}

\author{
Martha Morelos-Guzmán ${ }^{1,2}$, Larissa Minero-García,3*, José E. Jaramillo-Almaguer, \\ José F. Chávez-Carbajal', Carlos A. Arean-Martínez², Juan M. Vargas-Espinosa², \\ Martha E. Viveros-Sandovaß ${ }^{3}$, and Israel D. Campos-González $z^{5,6}$ \\ ${ }^{1}$ Department of Cardiovascular Imaging; ${ }^{2}$ Department of Internal Medicine and Cardiology; ${ }^{4}$ Centro de Investigación "Mario Alvizouri," Hospital \\ General "Dr. Miguel Silva;" 'Faculty of Medical and Biological Sciences "Dr. Ignacio Chávez," UMSNH, Department of Scientific Research; \\ ${ }^{5}$ Department of Research and Education, Clínica de Hemodiálisis NausLife; ${ }^{6}$ Department of Nephrology Research, Hospital General "Dr. Miguel \\ Silva." Morelia, Michoacán, Mexico
}

\begin{abstract}
Objective: The objective of the study was to analyze the clinical utility and feasibility of the multidetector cardiac tomography $(M D C T)$ in multi-parametric imaging assessment in atrial fibrillation (AF) patients. Materials and methods: This was a prospective case-control study in 84 subjects (54 AF subjects and 30 healthy subjects). Left atrial appendage (LAA) morphology was classified as: cactus, chicken wing, wind sock, and cauliflower. Intra-cardiac thrombus, stroke history, and $\mathrm{CH}_{2} D \mathrm{~S}_{2}-$ VASC scale were compared to cardiac MDCT atrial imaging assessment. Results: Left atrial ejection fraction (LAEF) and LAA ejection fraction (LAAEF) were lower in AF subjects $(p<0.001)$, left atrial volume index (LAVI) was higher in AF subjects $(p<0.001)$. An inverse correlation between LAEF and LAVI was found $(r=-0.38, p<0.001)$. Cauliflower LAA morphology frequency was higher in AF subjects, whereas cactus $L A A$ morphology frequency was higher in controls. Cauliflower $L A A$ morphology was associated with thrombus presence $(p<0.01)$ as well as a higher $\mathrm{CHA}_{2} D S_{2}-V A S c$ score. Flow velocity was lower in AF subject compared to controls $(p<0.001)$. Conclusion: MDCT is a novel, non-invasive, worldwide available method for an integral assessment in AF. Our results could improve precision, clinical utility, and risk stratification analysis in $A F$. Our proposal is to include this new method into the global cardiovascular and thrombotic risk assessment in AF patients.
\end{abstract}

Key words: Multidetector cardiac tomography. Atrial fibrillation. Atrial function. Left atrial appendage. Flow velocity.

\section{Resumen}

Objetivo: Analizar la utilidad clínica y la viabilidad de la tomografía cardíaca multidetector (TCMD) en la valoración de pacientes con fibrilación auricular (FA). Material y métodos: Estudio prospectivo de casos y controles en 84 sujetos (54 con FA y 30 controles). La morfología de la orejuela izquierda (OI) se clasificó en cactus, ala de pollo, manga de viento y coliflor. La presencia de trombo en la Ol, el antecedente de accidente cerebrovascular y la escala $\mathrm{CHA}_{2} D S_{2}$-VASc se compararon

Correspondence:

*Larissa Minero-García

E-mail: larissamg.gm@ hotmail.com
Date of reception: 06-02-2020

Date of acceptance: 04-04-2020

DOI: 10.24875/ACME.M21000174
Available online: $25-05-2021$

Arch Cardiol Mex (Eng). 2021;91(1):41-48 www.archivoscardiologia.com 2604-7063 / @ 2020 Instituto Nacional de Cardiología Ignacio Chávez. Published by Permanyer. This is an open access article under the CC BY-NC-ND license (http://creativecommons.org/licenses/by-nc-nd/4.0/). 
con parámetros obtenidos por TCMD. Resultados: La fracción de expulsión de la aurícula izquierda (FEAl) y la fracción de expulsión de la orejuela izquierda (FEOI) fueron más bajas en los individuos con $F A(p<0.001)$. El volumen indexado de la aurícula izquierda (VIAl) fue mayor en los pacientes con FA ( $p<0.001)$. Se observó una correlación inversa entre la FEAI y el VIAI $(r=-0.38, p<0.001)$. La morfología tipo coliflor fue la más frecuente en enfermos con $F A$, mientras que la de tipo cactus predominó en los controles. La de tipo coliflor fue la más relacionada con presencia de trombos $(p<0.01)$, así como una calificación $\mathrm{CHA}_{2} D S_{2}-V A S c$ alta. La velocidad de flujo fue menor en los pacientes con $F A(p<0.001)$. Conclusión: La TCMD es un método novedoso y no invasivo para una valoración integral en la FA. Los resultados de este estudio podrían mejorar la precisión, la utilidad clínica y el análisis de estratificación del riesgo en la FA. Los autores proponen incluir este nuevo método en la valoración integral del riesgo tromboembólico en pacientes con FA.

Palabras clave: Tomografía cardíaca multidetector. Fibrilación auricular. Función auricular. Orejuela izquierda. Velocidad de flujo

\section{Introduction}

Atrial fibrillation (AF) is the most common adult cardiac arrhythmia in clinical practice. At present, a global incidence of $1-2 \%$ is estimated in the general population, nearly 33.5 million people ${ }^{1}$. Around one out of every 5 strokes is due to AF; they are almost always serious and cause long-term disability or death ${ }^{2}$.

The $\mathrm{CHA}_{2} \mathrm{DS}_{2}$-VASc classification is a tool that is widely used to assess the risk of suffering an embolic episode, and it is essential to identify those patients who benefit from anticoagulant treatment ${ }^{3}$. Transthoracic echocardiogram constitutes a useful tool and is undoubtedly the basic evaluation technique in with patients AF; however, it has limited capacity for the detection of thrombi in the left atrium (LA) and left atrial appendage $(\mathrm{LAA})^{4}$. Transesophageal echocardiogram (TEE) is the imaging modality most widely used in patients with AF before pulmonary vein ablation procedures or in LAA percutaneous closure ${ }^{5}$. Recently, Nucifora et al. have proposed three-dimensional echocardiography for the assessment of LAA morphology ${ }^{6}$.

Cardiac magnetic resonance imaging (MRI) is an alternative non-invasive imaging modality in cases where TEE is not feasible, but its high cost and low availability difficult its use?

Current advances in cardiac multidetector computed tomography (MDCT) allow high spatial and temporal resolution, with three-dimensional images that enable non-invasive quantitative and multiparametric assessment of the LA and appendage. There are already comparative studies between TEE and MDCT in the identification of intracavitary thrombi, where MDCT showed better diagnostic accuracy. However, no comparison has been made of these two methods in patients with $\mathrm{AF}^{8}$. Left atrial volume index (LAVI) and left atrial ejection fraction (LAEF) have already been assessed with MDCT in patients with coronary artery disease $^{9}$ and in the healthy population ${ }^{10}$. Burrell et al. found an increase in LAVI in patients with $\mathrm{AF}$ in relation to a control group $(p<0.001)$. In addition, they pointed out that, in the presence of $A F$, there is LAA contractility and function decrease that manifests itself as a decrease in LAA flow and dilatation velocities ${ }^{11}$. Mügge et al. demonstrated that there is a correlation between LAA ejection fraction (LAAEF) decrease and thrombus formation on TEE in patients with $\mathrm{AF}^{12}$. Di Biase et al. classified LAA morphology in four categories: "chicken wing," which is the most common in the healthy population (48\%), followed by "cactus" $(30 \%)$, "windsock" $(19 \%)$, and "cauliflower" $(3 \%)^{13}$. Bosi et al. demonstrated, in anatomical models in vitro, that LAA morphology is related to blood flow hemodynamics and suggested that the lower the flow velocity, the higher the possibility of thrombotic episodes. These specialists found that the "cauliflower" morphology has the lowest flow velocity and that the "windsock" type is related to a higher flow velocity ${ }^{14}$.

At present, the most widely used method to quantify LAA flow velocity is TEE, although this method is invasive and entails certain complications. Yasuoka et al. conducted a study where they correlated TEE- and MDCT-obtained LAA flow velocity and found a good correlation between both methods ${ }^{15}$. A recent meta-analysis that included 19 studies with 2,955 patients showed a sensitivity of $96 \%$, specificity of $92 \%$, and a robust negative predictive value of MDCT for the detection thrombi $(96-100 \%)^{16}$. Wu et al. proposed that patients without evidence of thrombi on MDCT do not require TEE for confirmation ${ }^{17}$. Furthermore, MDCT has recently been shown to be superior to transthoracic and transesophageal echocardiography for the assessment of LA size, an important predictor of $A F$ recurrence ${ }^{18}$.

MDCT enables full assessment in patients with AF; so far, there are no studies simultaneously evaluating LAEF, LAAEF, LA volume index and LAA, LAA morphology, LAA flow velocity, and presence of thrombus 


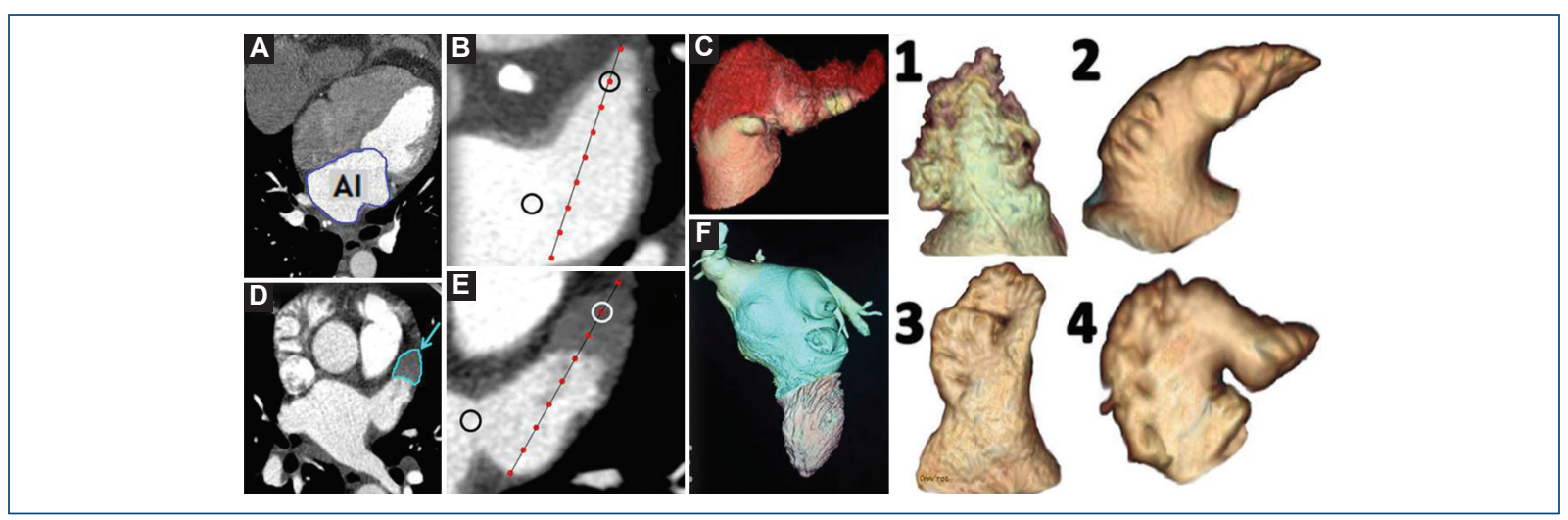

Figure 1. Multidetector computed tomography cardiac parameters. A: Left atrial (LA) volume index. B: Left atrial appendage (LAA) flow velocity in a patient of the control group. C: LAA thrombus three-dimensional reconstruction. D: LAA thrombus (arrow). E: LAA flow velocity in a patient of the atrial fibrillation group. F: LA aneurysm threedimensional reconstruction. Three-dimensional reconstruction of LAA different types of morphology: 1) cauliflower, 2) windsock, 3) cactus, and 4) chicken wing.

in LAA by MDCT. This multiparametric assessment offers more information to the doctor for optimizing treatment and allows thromboembolism risk re-stratification in patients with AF, which could modify these patients' quality of life and prognosis. This study will open new horizons in the research related to this topic, since medical publications are scarce and it is foreseeable that it will serve as a reference for future research.

\section{Materials and Methods}

This was a prospective study carried out between March 1, 2018, and April 1, 2019, at Hospital General "Dr. Miguel Silva" in the city of Morelia, Michoacán, Mexico. The study received approval from the ethics committee with registration number Conbioética-16-CEI-004-20161212, and included a total of 84 patients. Out of them, 54 had been diagnosed with AF and 30 constituted the control group. The latter group was made up of patients who underwent MDCT for clinical suspicion of ischemic heart disease and did not suffer from AF; these controls had some risk factor (high blood pressure (HBP), diabetes mellitus (DM), dyslipidemia, smoking, alcoholism, or obesity).

Both groups were matched by age and gender. All patients were evaluated using the $\mathrm{CHA}_{2} \mathrm{DS}_{2}$-VASc scale to calculate thromboembolic risk. Weight and height were included to calculate body mass index (BMI), as well as other clinical variables such as smoking, alcoholism, HBP, DM, dyslipidemia, and a history of cerebrovascular event or transient ischemic attack
(CVE/TIA). Patients with valvular heart disease, cardiomyopathies, congenital diseases, autoimmune diseases, kidney failure, or allergy to contrast medium were excluded from the study. All patients underwent MDCT to obtain the images described by Gweon et al. ${ }^{19}$ Thirty-two and 64-slice CT scanners (General Electric) were used and contrast medium (Iopamiron ${ }^{\circledR} 370$ ) was administered. Beta-blocker with metoprolol was orally administered before the test with the purpose to achieve a heart rate of 60 beats/min. LA and LAA three-dimensional structures were reconstructed with the volume rendering processing technique to assess $L A$ and $L A A$ volume index and expulsion fraction. LA and LAA flow velocity and volume variables were calculated, as well as the presence of thrombi. LAA morphology was evaluated in multiplanar reconstructions and classified by two cardiac imaging experts, who were blinded to patient clinical data.

A cardiovascular imaging specialist performed the analysis of the obtained images using a GE workstation (CardiolQ Express, version 8; General Electric Medical Systems). To determine LAA flow velocity, a two-dimensional image of said structure was obtained and, subsequently, two different points were determined in the LAA (distal and proximal) (Fig. 1B and E); the proximal point was obtained at the center of the LAA orifice, while the distal point was the 7/8 point of a straight line drawn from the flexion point of LAA posterior wall to LAA most anterior distal point. Once both these values were obtained, the ratio of the distal point to the proximal point was calculated, and the result was a ratio in Hounsfield units. Yasuoka previously described and 
approved this method ${ }^{15}$, LAA morphology was evaluated according to the Di Biase classification ${ }^{13}$, and LAA volume index was calculated based on the LAA maximum and minimum volumes that were determined with the volume rendering processing technique. LAAEF was calculated with the following formula: $E F=\left(V_{\max }\right.$ $\left.\left.-\mathrm{V}_{\min }\right) / \mathrm{V}_{\text {max }}\right)$, based on the Boucebci method ${ }^{20}$.

\section{Statistical analysis}

A one-way analysis of variance or Kruskal-Wallis $\mathrm{H}$-test with Dunn post hoc was used for continuous variables, such as age, weight, body mass index, and all radiological quantitative characteristics grouped by type of LAA, both in the AF group and in controls; statistical contrast of averages was applied between AF and controls and, within the AF group, between both AF subgroups (paroxysmal vs. permanent), with oneand two-tailed Student's t-test, respectively; the linear correlation coefficient product of Pearson's moment was analyzed for both groups together with LAA flow velocity with respect to the other quantitative variables, and the Chi-square test or Fisher's exact $F$ test were applied for the contrast of frequencies in $2 \times 2$ Latin square; in all cases, the statistically significant likelihood of alpha error was $<0.05$, according to the SigmaPlot 12.5 (CR) 2003-2013 program.

\section{Results}

Eighty-four individuals were analyzed, 54 of them with AF: 21 (38.9\%) with paroxysmal AF and 33 (61.1\%) with permanent AF. Mean age in the AF group was 67 \pm 14 years and $55.6 \%$ corresponded to males. Table 1 describes both groups' clinical characteristics. An inverse correlation was found between LAEF and age $(r=-0.43, p<0.001)$, as well as with LAAEF $(r=-0.33$, $p<0.001)$.

Cauliflower-type morphology was the most common in the AF group (33.3\%), while cactus type predominated in the controls $(37 \%)$. In the LAVI and LAEF analysis, significant differences were found between both groups $(p<0.001)$, and differences with LAAEF and LAA flow velocity were also documented between cases and controls $(p<0.001)$.

The parameters evaluated by MDCT are presented in table 2. LAA flow velocity was lower in the AF group with regard to the control group $(0.34 \pm 0.16 \mathrm{~cm} / \mathrm{s}$ vs. $0.87 \pm 0.08 \mathrm{~cm} / \mathrm{s})(p<0.001)$. In patients with AF, LAA flow velocity was slower in those who had an LAA thrombus, but no statistical difference was found with
Table 1. Control and atrial fibrillation groups clinical characteristics

\begin{tabular}{|c|c|c|c|}
\hline & $\begin{array}{l}\text { Control } \\
\text { group } \\
(n=30)\end{array}$ & $\begin{array}{l}\text { AF group } \\
(n=54)\end{array}$ & $\mathbf{p}$ \\
\hline Age (years) & $54.83 \pm 15$ & $67.43 \pm 14$ & $<0.001$ \\
\hline Male gender & $53.3 \%$ & $55.6 \%$ & $\mathrm{~N} / \mathrm{S}$ \\
\hline BMI & $27.9 \pm 5$ & $28 \pm 5$ & $\mathrm{~N} / \mathrm{S}$ \\
\hline High blood pressure & $43.3 \%$ & $46.3 \%$ & $\mathrm{~N} / \mathrm{S}$ \\
\hline Dyslipidemia & $23.3 \%$ & $24.1 \%$ & $\mathrm{~N} / \mathrm{S}$ \\
\hline Diabetes mellitus & $6.7 \%$ & $25.9 \%$ & $<0.001$ \\
\hline Smoking & $36.7 \%$ & $31.5 \%$ & $\mathrm{~N} / \mathrm{S}$ \\
\hline Alcoholism & $23.3 \%$ & $22.2 \%$ & $\mathrm{~N} / \mathrm{S}$ \\
\hline History of CVE/TIA & $0 \%$ & $33.3 \%$ & $<0.001$ \\
\hline Anticoagulation & $0 \%$ & $42.6 \%$ & $<0.001$ \\
\hline $\begin{array}{l}\text { Antiplatelet } \\
\text { aggregation }\end{array}$ & $0 \%$ & $53.7 \%$ & $<0.001$ \\
\hline $\begin{array}{l}\text { Type of AF } \\
\text { Paroxysmal AF } \\
\text { Permanent AF }\end{array}$ & & $\begin{array}{l}38.9 \% \\
61.1 \%\end{array}$ & \\
\hline CHADS2 scale & & $1.7 \pm 1.2$ & \\
\hline CHA2DS2-VASc scale & & $2.8 \pm 1.7$ & \\
\hline
\end{tabular}

BMI: Body Mass Index; CVE: cerebrovascular episode; TIA: transient ischemic attack; AF: atrial fibrillation.

regard to those who did not suffer thrombus $(0.32 \pm$ 0.14 vs. $0.35 \pm 0.17 \mathrm{~cm} / \mathrm{s} ; p=N S$ ) (Fig. 2).

The AF group analysis found that LAVI was higher in patients who had LAA thrombus in comparison with those who did not $\left(82 \pm 32.7\right.$ vs. $48.3 \pm 14.9 \mathrm{ml} / \mathrm{m}^{2}$, respectively; $p<0.001$ ). LAEF was lower in those who showed LAA thrombus versus individuals who did not $(14.1 \pm 7.9$ vs. $23.6 \pm 11.8 \% ; p<0.001)$; the same results were obtained for LAAEF (11.6 \pm 6.2 vs. $18.2 \pm 9 \%$; $p=0.002$ ) (Fig. 3).

Some important differences in LAA morphology were identified in the AF group; the cauliflower-type morphology was the most common in patients in whom LAA thrombus was detected ( $p \leq 0.01$ ); subsequently, the cauliflower-type morphology was compared with forms other than this in patients with LAA thrombus, and 5.9 versus $7.4 \%$ ( $p<0.001)$; OR, 4.7 ; and $95 \% \mathrm{Cl}, 1.7-12.4$, respectively, were observed. The windsock-type morphology was associated with absence of thrombus $(p<0.05)$ (Fig. 4). A high versus low to moderate $\mathrm{CHA}_{2} \mathrm{DS}_{2}$-VASc score was compared regarding the 


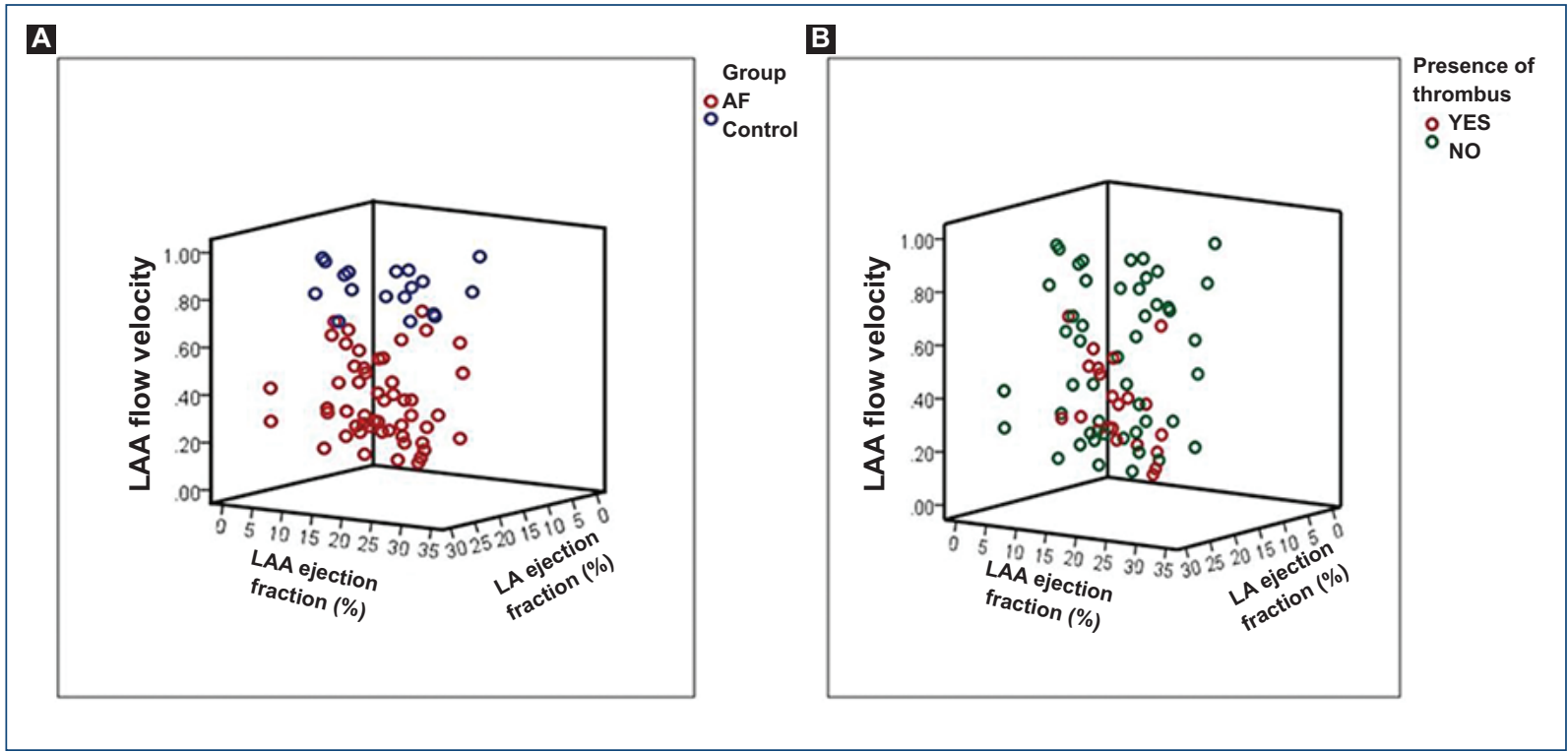

Figure 2. A: Left atrial appendage (LAA) flow velocity in subjects with atrial fibrillation (AF) in comparison with controls. B: LAA flow velocity in patients with AF with and without thrombus. AF group versus control group ( $p<0.01$; Student's t-test).

Table 2. Variables on cardiac multidetector computed tomography

\begin{tabular}{|c|c|c|c|}
\hline & $\begin{array}{l}\text { Control } \\
\text { group } \\
(n=30)\end{array}$ & $\begin{array}{c}\text { AF group } \\
(\mathrm{n}=54)\end{array}$ & $\mathbf{p}$ \\
\hline Calcium score (AU) & $10.8 \pm 24$ & $59.26 \pm 142$ & $\mathrm{~N} / \mathrm{S}$ \\
\hline LAVI $\left(\mathrm{mL} / \mathrm{m}^{2}\right)$ & $40.3 \pm 9$ & $67.3 \pm 27.4$ & $<0.001$ \\
\hline LA Vol $\mathrm{I}_{\max }(\mathrm{mL})$ & $75.2 \pm 20$ & $116.4 \pm 49$ & $<0.001$ \\
\hline $\mathrm{LA} \mathrm{Vol} \mathrm{I}_{\min }(\mathrm{mL})$ & $53.4 \pm 19.3$ & $96.6 \pm 42.8$ & $<0.001$ \\
\hline LAEF $(\%)$ & $29.8 \pm 12.5$ & $16.2 \pm 7.7$ & $<0.001$ \\
\hline LAA Vol $\mathrm{max}_{\max }(\mathrm{mL})$ & $10.8 \pm 4.4$ & $12.6 \pm 5.7$ & $\mathrm{~N} / \mathrm{S}$ \\
\hline LAA Vol $\mathrm{Imin}_{(\mathrm{mL})}$ & $8.5 \pm 3.7$ & $11 \pm 5.4$ & $<0.001$ \\
\hline LAAEF (\%) & $21.1 \pm 9$ & $13.87 \pm 7.7$ & $<0.001$ \\
\hline LAAFV (HU ratio) > & $0.72 \pm 0.20$ & $0.34 \pm 0.16$ & $<0.001$ \\
\hline $\begin{array}{l}\text { LAA morphology } \\
\text { Cauliflower } \\
\text { Windsock } \\
\text { Chicken wing } \\
\text { Cactus }\end{array}$ & $\begin{array}{c}20.0 \% \\
26.7 \% \\
16.7 \% \\
37 \%\end{array}$ & $\begin{array}{l}33.3 \% \\
27.8 \% \\
16.7 \% \\
22.2 \%\end{array}$ & $\mathrm{~N} / \mathrm{S}$ \\
\hline Thrombus (\%) & $0 \%$ & $42.5 \%$ & $<0.001$ \\
\hline
\end{tabular}

LAVI, left atrial volume index; LA Vol : left atrial maximum volume; LA Vol : left atrial minimum volume; LAEF: left atrial ejection fraction; LAA Vol $\left.\right|_{\max }$ : left atrial appendage maximum volume; LAA Vol ${ }_{\text {min }}$ : left atrial appendage minimum volume; LAAEF: left atrial appendage ejection fraction; LAAFV: left atrial appendage flow velocity.

presence of LAA thrombus, with thrombus being found in 16.7 versus $9.3 \%(p=0.056)$; OR, $2.4 ; 95 \% \mathrm{Cl}, 0.93$ 6.2 , respectively.
In the AF group, 23 patients (42.6\%) received anticoagulant treatment. Of these individuals, 10 (43.5\%) had LAA thrombus, while among those who were not anticoagulated, $13(41.9 \%)$ had LAA thrombus $(p<0.01)$.

\section{Discussion}

At present, TTE and TEE are the most commonly used imaging modalities for risk stratification assessment in patients with AF; however, the latter is an invasive method that entails potential risks ${ }^{20}$. TEE is considered the reference standard ${ }^{2}$ for thrombi identification in AF. MDCT is a non-invasive method that allows multi-parametric assessment in patients with $\mathrm{AF}$; however, its role is not well defined, as there are few studies about it and, to date, no publication has comprehensively evaluated patients with AF using MDCT. In this study population, a total of 84 patients were included, and AF patients mean age was 67 years versus 55 years in the control group. Despite not having the same sample number for both groups, this parameter was significant $(p<0.001)$ and agrees with publications where AF average age is around the seventh decade of life. This is important, because there is an increase in $\mathrm{AF}$ as age advances ${ }^{21}$. Baseline characteristics between both groups of this study population (smoking, alcoholism, HBP, and dyslipidemia) showed no significant differences.

As for the treatment of patients with AF, only $42.6 \%$ (23 patients) were identified to be on anticoagulant 


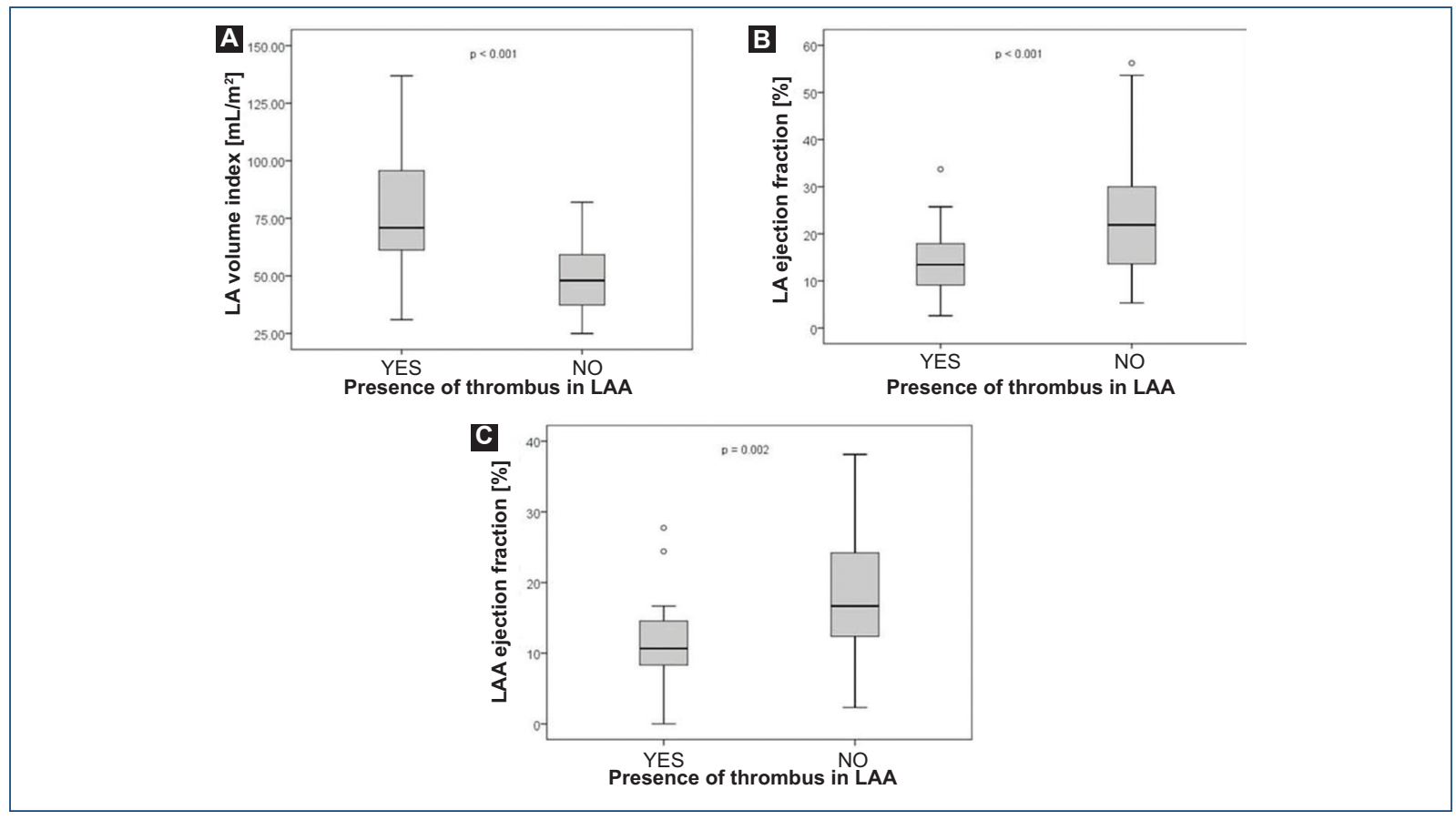

Figure 3. Differences between left atrial volume index and left atrial and left atrial appendage ejection fractions in patients with and without thrombus (Student's t-test).

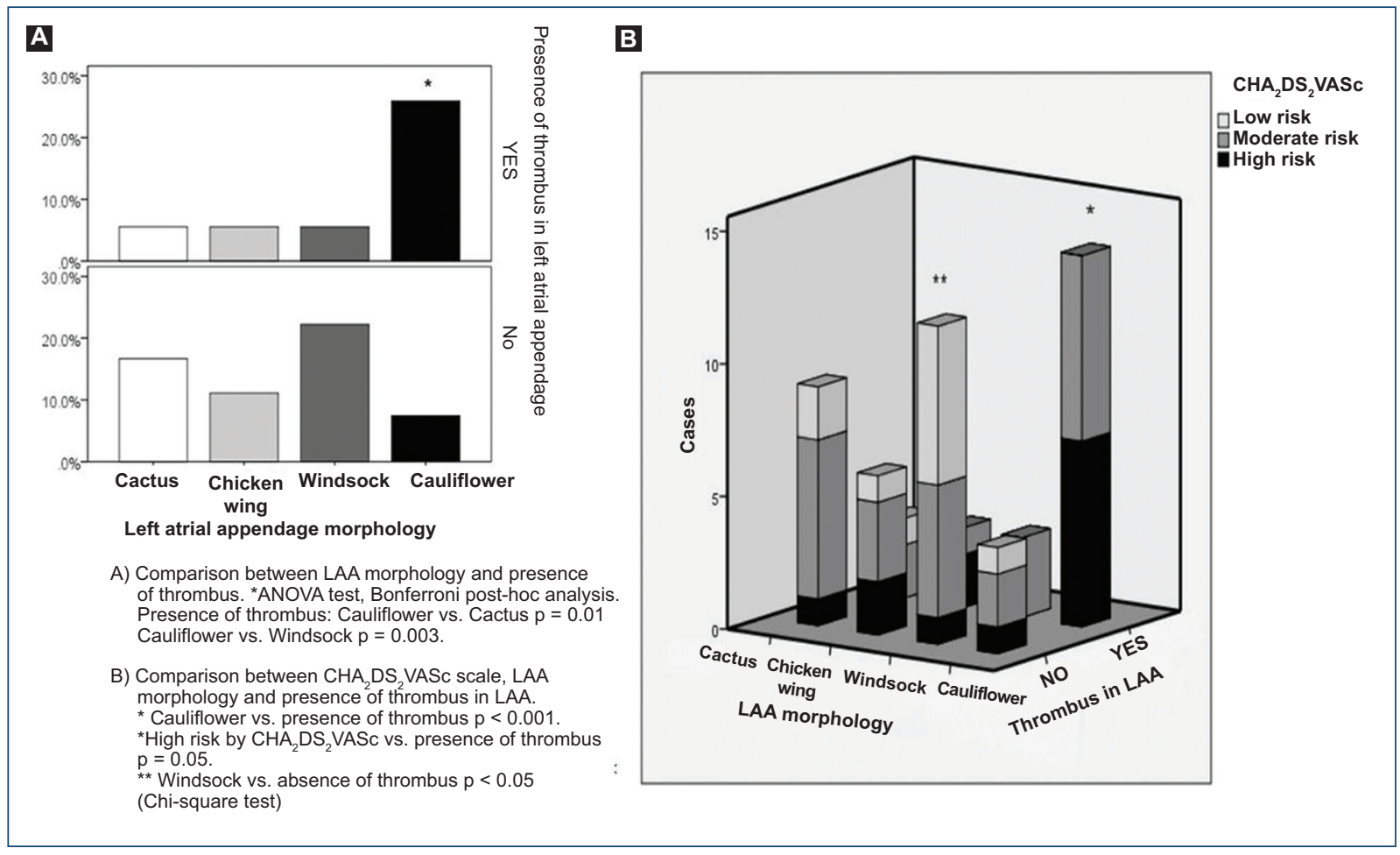

Figure 4. Comparison between left atrial appendage different morphology types and the $\mathrm{CHA}_{2} \mathrm{DS}_{2}$-VASc scale.

treatment, while $53.7 \%$ (29 patients) were taking platelet aggregation inhibitors. This probably was due to the fact that a large percentage of these patients attended the outpatient cardiology clinic for the $1^{\text {st }}$ time, 
where diagnosis was established and treatment was started. LA dilation is a marker of adverse cardiovascular events, such as stroke, heart failure, or even death. Pavón et al. mention that LAVI should be a systematic measure of atrial growth assessment due to its high sensitivity and specificity. In addition, it reflects an elevation of left ventricular filling pressure and is therefore an important predictor of poor prognosis in patients with $\mathrm{AF}^{22}$. In this study, the authors found that LAVI was markedly higher in the AF population with regard to the control group, even though controls also recorded altered LAVI values (40.3 \pm $\left.9 \mathrm{ml} / \mathrm{m}^{2} ; \mathrm{p}<0.001\right)$. This is important, since AF causes atrial growth per se, which favors an increase in filling pressure and thereby a self-perpetuating cycle of arrhythmia is established. Consequently, restoring sinus rhythm prevents and reverses atrial dilatation in these patients, by normalizing filling pressures and cardiac output, which improves patient prognosis ${ }^{22}$. As for LAA morphology, no significant differences between both groups were identified in this study, probably due to the small size of the sample. However, the "cauliflower" morphology was observed to be the most closely related to the presence of LAA thrombi. The "windsock" and "chicken wing" types were found to be the least associated with the presence of thrombi. These results are consistent with those of Bosi et al., who found that "cauliflower"-type LAA had a lower flow velocity than the rest of morphologies and, although this is a study in anatomical models, it provides an important analysis on thrombogenesis pathophysiology in $L_{A A}{ }^{14}$. In patients with $A F$, a marked decrease in LAA flow velocity was identified versus controls $(p<0.001)$. This can be explained by the anatomical differences of each appendage (angles of the main lobes relative to the ostium, number of trabeculations, and number of lobes). The "chicken wing" shape reveals an obtuse angle that allows higher flow velocity (faster emptying); some studies have described that it is the least related to the presence of thrombi. "Cauliflower"-type LAAs have an acute angle with regard to the ostium, which makes its emptying difficult and, therefore, this shape is most times associated with thrombi formation ${ }^{23}$. As for LA and LAAEF in the general population, an inverse correlation has been demonstrated (the older the age, the lower the ejection fraction); Handke et al. ${ }^{24}$ found similar results. In this study, ejection fraction was observed to be lower in the AF group in comparison with controls $(p<0.001)$ and, within the AF group, ejection fraction was lower when there were LAA thrombi $(p<0.001)$; this can considered a poorer prognosis criterion in this group of patients. Another analyzed clinical variable was the $\mathrm{CHA}_{2} \mathrm{DS}_{2}$-VASc scale assessment, where a high value was identified to predominate in the population with $\mathrm{AF}$, and the type that was associated with this parameter was that of "cauliflower." Furthermore, in the AF group, 18 patients had a history of a vascular event (CVE or TIA). In in this subgroup of patients, the "cauliflower" type predominated over the others, which once again supports the theory that this atrial appendage morphology is the most thrombogenic.

The main limitation of the study is the same that has been faced by most authors who have published on this subject, that is, sample size. Nevertheless, in this preliminary investigation, the authors recorded significant results. One of the advantages for the performance of this study was the integration of a multidisciplinary team made up by clinical cardiologists, cardiovascular imaging specialists, and radiologists.

At presdent, TEE is carried to rule out the presence of thrombi in LAA before invasive AF procedures for therapeutic purposes, such as LAA occlusion or ablation of the pulmonary veins; however, it is important mentioning that this method is invasive and there is risk of potential complications with the procedure, in addition to a higher cost in comparison with MDCT ${ }^{20}$. MDCT is a non-invasive method that is currently used. Some disadvantages of this method in relation to the others include costs, use of contrast medium and exposure to radiation. Nevertheless, MDCT has in its favor a better spatial and temporary resolution in comparison with other methods, which is important in this study for the correct determination of atrial appendage morphology. Moreover, with the new CT scanners, the radiation dose is increasingly lower (3-8 mSv) ${ }^{25}$.

This study shows that patients with AF may require, along with a clinical evaluation with the $\mathrm{CHA}_{2} \mathrm{DS}_{2}$-VASc scale, electrocardiogram, Holter monitoring and echocardiogram, plus a MDTC, since it is possible for information such as LAA morphology, atrial function, and LAA parameters to be obtained, as well as flow velocity, which appear to play an important role in LAA thrombogenesis. After these parameters are obtained through this method, selecting the most convenient treatment for each patient is possible, which results in an important and novel application, and selection of patients that are suitable for LAA occlusion.

This study descriptively details the findings with regard to MDCT, and determining its sensitivity, specificity, and false and negative positive rate was not possible given that it was not compared with the current 
reference standard, that is, TEE. It is important for these methods to be compared in future investigations and these parameters to be determined, since publications on this subject are scarce.

\section{Conclusions}

MDCT is a non-invasive method that allows a comprehensive, useful, and accurate assessment for thromboembolic risk re-stratification in patients with $\mathrm{AF}$, and provides relevant information to make decisions in the treatment and prognosis of patients with AF. This protocol should be implemented in the approach to the study of patients with AF.

\section{Funding}

This research did not receive any specific subsidy from agencies in the public, commercial, or non-profit sectors.

\section{Conflicts of interest}

The authors declare that they have no conflicts of interest.

\section{Ethical disclosures}

Protection of human and animal subjects. The authors declare that the procedures that were followed adhered to the ethical standards of the responsible committee for experimentation in human beings and were in agreement with the World Medical Association and the Declaration of Helsinki.

Confidentiality of data. The authors declare that they have followed the protocols of their work center on the publication of patient data.

Right to privacy and informed consent. The authors have obtained informed consent from the patients or subjects referred to in the article. This document is in the possession of the corresponding author.

\section{References}

1. Morin DP, Bernard ML, Madias C, Rogers PA, Thihalolipavan S, Estes NAM. The state of the art. Mayo Clin Proc. 2016;91(12):1778-810.

2. National Collaborating Centre for Chronic Conditions (UK). Chronic heart failure: National Clinical Guideline for Diagnosis and Management in Primary and Secondary Care. London: Royal College of Physicians (UK); 2003 (National Institute for Health and Clinical Excellence: Guidance) Disponible en: http://www.ncbi.nlm.nih.gov/books/NBK65609

3. Coppens M, Eikelboom JW, Hart RG, Yusuf S, Lip GYH, Dorian P, et al. The CHA2DS2-VASc score identifies those patients with atrial fibrillation and a CHADS2 score of 1 who are unlikely to benefit from oral anticoagulant therapy. Eur Heart J. 2013;34(3):170-6.
4. Aschenberg W, Schlüter M, Kremer P, Schröder E, Siglow V, Bleifeld W Transesophageal two-dimensional echocardiography for the detection of left atrial appendage thrombus. J Am Coll Cardiol. 1986;7(1):163-6.

5. Sallach JA, Puwanant S, Drinko JK, Jaffer S, Donal E, Thambidorai SK, et al. Comprehensive left atrial appendage optimization of thrombus using surface echocardiography: The CLOTS Multicenter Pilot Trial. J Am Soc Echocardiogr. 2009;22(10):1165-72

6. Nucifora G, Faletra FF, Regoli F, Pasotti E, Pedrazzini G, Moccetti T, et al. Evaluation of the Left atrial appendage with real-time 3-dimensional transesophageal echocardiography: implications for catheter-based left atrial appendage closure. Circulation Cardiovasc Imaging. 2011;4(5):514-23.

7. Rathi VK, Reddy ST, Anreddy S, Belden W, Yamrozik JA, Williams RB et al. Contrast-enhanced CMR is equally effective as TEE in the evaluation of left atrial appendage thrombus in patients with atrial fibrillation undergoing pulmonary vein isolation procedure. Heart Rhythm. 2013;10(7):1021-7.

8. Vázquez de Prada JA, Pérez de Isla L. Técnicas de imagen en la fibrilación auricular. Rev Esp Cardiol Supl. 2016;16:20-4.

9. Stojanovska J, Cronin P, Patel S, Gross BH, Oral H, Chughtai $\mathrm{K}$, et al Reference normal absolute and indexed values from ecg-gated mdct: left atrial volume, function, and diameter. Am J Roentgenol. 2011;197(3):631-7.

10. using 64-slice mdct to assess right atrial appendage anatomy and function at sinus rhythm and atrial fibrillation: a comparative study with the left atrial appendage and thrombus formation implications. J Cardiovasc Comp Tomograp. 2008;2:S18.

11. Burrell LD, Horne BD, Anderson JL, Muhlestein JB, Whisenant BK. Usefulness of left atrial appendage volume as a predictor of embolic stroke in patients with atrial fibrillation. Am J Cardiol. 2013;112(8):1148-52.

12. Mügge A, Kühn H, Nikutta $P$, Grote J, G. López JA, Daniel WG. Assessment of left atrial appendage function by biplane transesophageal echocardiography in patients with nonrheumatic atrial fibrillation: identification of a subgroup of patients at increased embolic risk. J Am Coll Cardiol. 1994;23(3):599-607.

13. Di Biase L, Santangeli P, Anselmino M, Mohanty P, Salvetti I, Gili S, et al. Does the left atrial appendage morphology correlate with the risk of stroke in patients with atrial fibrillation? J Am Coll Cardiol. 2012:60(6):531-8.

14. Bosi GM, Cook A, Rai R, Menezes LJ, Schievano S, Torii R, et al. Computational fluid dynamic analysis of the left atrial appendage to predict thrombosis risk. Front Cardiovasc Med. 2018:5:34

15. Yasuoka R, Kurita T, Kotake Y, Akaiwa Y, Hashiguchi N, Motoki K, et al. A novel method to estimate blood flow velocity in the left atrial appendage using enhanced computed tomography: role of Hounsfield unit density ratio at two distinct points within the left atrial appendage. Heart Vessels. 2017;32(7):893-901.

16. Romero J, Husain SA, Kelesidis I, Sanz J, Medina HM, García MJ. Detection of left atrial appendage thrombus by cardiac computed tomography in patients with atrial fibrillation: a meta-analysis. Circ Cardiovasc Imaging. 2013;6(2):185-94.

17. Wu X, Wang C, Zhang C, Zhang Y, Ding F, Yan J. Systematic review/ meta-analysis computed tomography for detecting left atrial thrombus: a meta-analysis. Arch Med Sci. 2012;6:943-51.

18. Hof I, ArbabnZadeh A, Scherr D, Chilukuri K, Dalal D, Abraham T, et al. Correlation of left atrial diameter by echocardiography and left atrial volume by computed tomography. J Cardiovasc Electrophysiol. 2009;20(2):159-63.

19. Gweon HM, Kim SJ, Kim TH, Lee SM, Hong YJ, Rim S-J. Evaluation of left atrial volumes using multidetector computed tomography: comparison with echocardiography. Korean J Radiol. 2010;11(3):286.

20. Boucebci S, Pambrun T, Velasco S, Duboe P-O, Ingrand P, Tasu J-P. Assessment of normal left atrial appendage anatomy and function over gender and ages by dynamic cardiac CT. Eur Radiol. 2016;26(5):1512-20.

21. Christiaens L, Lequeux B, Ardilouze P, Ragot S, Mergy J, Herpin D, et al. A new method for measurement of left atrial volumes using 64-slice spiral computed tomography: comparison with two-dimensional echocardiographic techniques. Int J Cardiol. 2009;131(2):217-24

22. Pavón-Jiménez R, García-Medina D, Villagómez-Villegas D. ¿Debemos medir siempre el tamaño de la aurícula izquierda en pacientes con fibrilación auricular? ¿Cómo? ¿Por qué? Cardiocore. 2010;45(3):93-5.

23. Mulder MJ, Götte MJW, Allaart CP. Left atrial appendage morphology in atrial fibrillation: Do we prefer chicken wing or cauliflower? J Cardiovasc Comput Tomogr. 2019;:S1934592519301662.

24. Handke M, Harloff A, Hetzel A, Olschewski M, Bode C, Geibel A. Left atrial appendage flow velocity as a quantitative surrogate parameter for thromboembolic risk: determinants and relationship to spontaneous echocontrast and thrombus formation-a transesophageal echocardiographic study in 500 patients with cerebral ischemia. J Am Soc Echocardiogr. 2005;18(12):1366-72.

25. Izeta-Gutiérrez AC, Ojeda-Delgado JL, Mendizábal-Méndez AL, Valdez-Becerril G, Millán-Contreras S, Gutiérrez-García Z, et al. Tomografía computada de corazón, una herramienta invaluable. Rev Sanid Mil. 2014;68(1):36-41. 